\title{
Vibrational Electron Energy Loss Spectroscopy of Astrosilicates
}

\author{
Rhonda M Stroud ${ }^{1}$, Maureen J. Lagos ${ }^{2}$ and Philip E. Batson ${ }^{2}$ \\ 1. Materials Science and Technology Division, Naval Research Laboratory, Washington, DC, USA \\ 2. Department of Physics and Materials Science, Rutgers University, Piscataway, NJ, USA.
}

Vibrational spectroscopy in the electron microscope [1] offers the possibility of exploring the infrared (IR) optical properties of materials at the nanometer scale, in direct correlation with structural and elemental properties. One potentially important application of vibrational electron energy loss spectroscopy (VEELS) is mineralogical analysis of astrophysical materials. Astronomers monitor the evolution of stars through IR spectroscopy of the condensed nanoscale dust. Determination of the mineralogy of planetary bodies, including the Moon, Mars, asteroids, and comets is also all performed with remote IR sensing. Unique interpretations of the astronomical and remote sensing spectra are difficult, in part due to the lack of reference spectra from actual cosmic dust particles. While there is a great deal of information about the mineralogy of astrosilicates already known from detailed electron microscopy studies, the small grain size (10s to 100s of $\mathrm{nm}$ ) of much of the dust has hindered direct measurement of the IR properties of individual grains by conventional optical spectroscopy.

In order to test the utility of VEELS as a tool for astromineralogy, we measured a series of sub-micron silicates in a cross section extracted from a primitive meteorite. The cross section was prepared with the FEI Helios G3 FIB at the Naval Research Laboratory (NRL). High angle annular dark field (HAADF) imaging and energy dispersive x-ray (EDX) spectroscopy studies of the section were performed with the Nion UltraSTEM 200-X at NRL. Vibrational EELS data were obtained at Rutgers University with the Nion HERMES and customized spectrometer. The HERMES was operated at $60 \mathrm{kV}$, with a $10 \mathrm{meV}$ monochromated probe, a current at the sample of $\sim 10 \mathrm{pA}$, and an energy dispersion of $0.7 \mathrm{meV} /$ channel. Spectra from the olivine and GEMS grain were recorded as a series of 30 summed $1 \mathrm{sec}$ acquisitions, and from the amorphized olivine and altered GEMS grains as 30 summed 2 sec acquisitions. Spectra shown in: Fig. 2 are processed only for instrumental artifacts; in Fig. 3 (left) are converted to wavelength scale, and (Fig. 3 right) with the subtraction background empirically estimated as a continuum, and smoothed with 5 to $10 \mathrm{pt}$ adjacent averaging.

The measured astrosilicate VEELS data show good agreement with the published IR spectra for crystalline and amorphous silicates [2]. The Si-O stretch (130 meV) and Si-O bend (60 meV) modes, known in astronomy as the $10 \mu \mathrm{m}$ and $18 \mu \mathrm{m}$ features, are clearly observed. The olivine grain spectrum resolves the expected split of the $10 \mu \mathrm{m}$ feature into sharp peaks at $9.9 \mu \mathrm{m}$ and $11.2 \mu \mathrm{m}$. The amorphous GEMS grains have broader Si-O stretch features and a less prominent Si-O bend feature. The feature at $200 \mathrm{meV}(6.6 \mu \mathrm{m})$ in the altered GEMS and amorphized olivine spectra is likely associated with water absorption from direct hydration of silicates and/or through $\mathrm{H}$ passivation of radiationinduced defects [3]. While more rigorous spectrum processing methods will be needed to make quantitative analyses, it is clear that VEELS can be an important method for relating actual individual astronomical nanoparticles to remote IR spectra from specific astrophysical environments.

References:

[1] O Krivanek et al., Nature 514 (2014), p. 209. 
[2] FJ Molster, LBFM Waters and AGGM Tielens, Astron. \& Astrophys. 382 (2002), p. 222.

[3] P Lucey et al., Lunar and Planetary Science XLVIII (2017), Abstract \#1314.

[4] RMS acknowledges funding from NASA on grants NNH16AC42I, and NNA14AA82I, and the loan of the meteorite sample (LAP 02342) from the MWG, through L. Nittler and J. Trigo-Rodriguez.

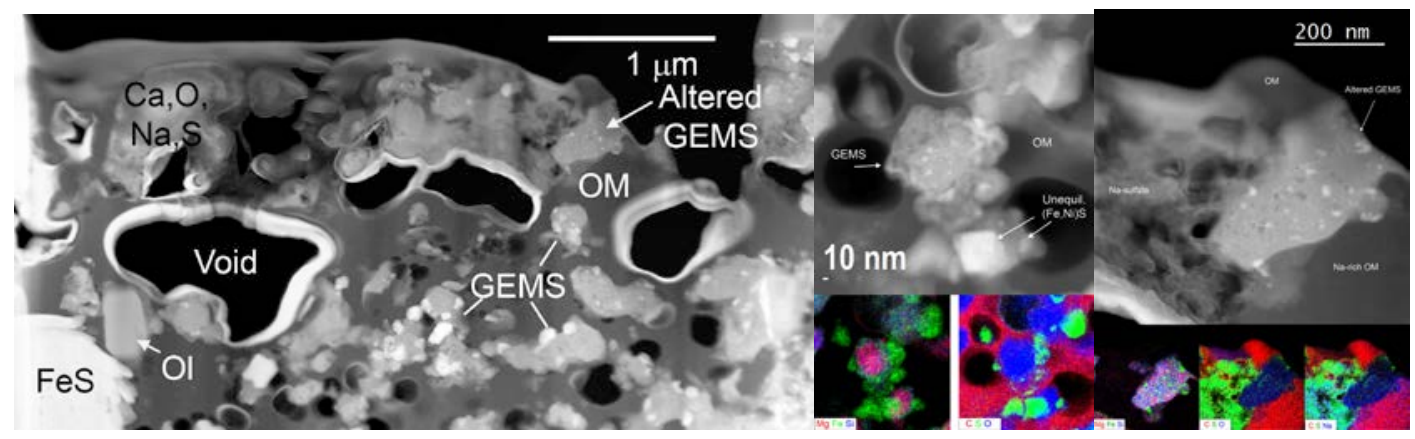

Figure 1. (Left) HAADF image of a primitive meteorite containing three common classes of astrosilicates: Mg-rich olivine (Ol), Mg-rich silicate glass with embedded Fe metal and sulfides (GEMS), and GEMS altered by aqueous fluids (altered GEMS), surrounded by organic matter (OM). (Middle) HAADF image and composite EDX maps of a GEMS grain, (Right) HAADF image and composite EDX maps of an altered GEMS grain.
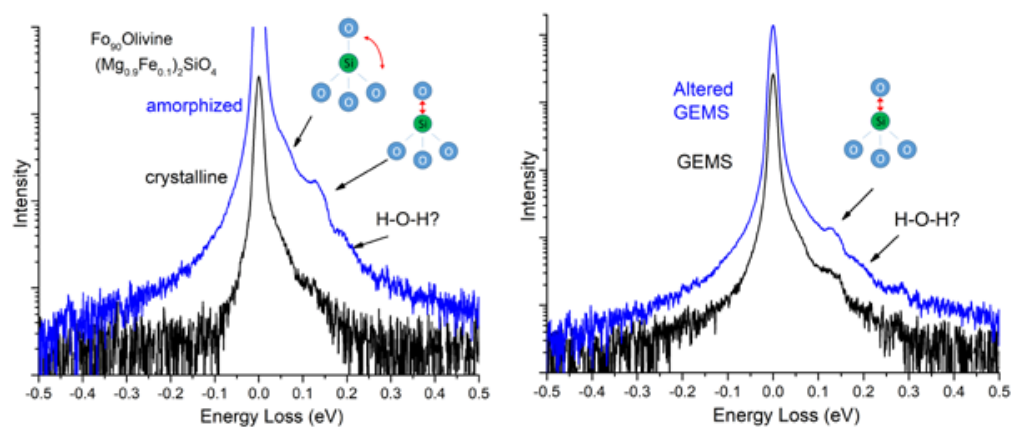

Figure 2. (Left) Raw VEEL spectra of the olivine (Fig. 1) before and after electron beam amorphization. The crystalline grain shows sharp Si-O stretch modes at $113 \mathrm{meV}$ and $128 \mathrm{meV}$, and a weaker Si-O bend mode feature near $60 \mathrm{meV}$. Beam damage to the lattice broadens the stretch modes, and introduces a new feature near $200 \mathrm{meV}$, likely associated with a H-O-H bend mode. (Right) VEEL spectra of GEMS and altered GEMS grains (Fig. 1). The GEMS grain shows a weak Si-O bend feature and prominent Si-O stretch feature, whereas the altered GEMS shows a shift of the Si-O stretch to lower energy, and the emergence of the $200 \mathrm{meV} \mathrm{H-O}-\mathrm{H}$ bend mode feature.
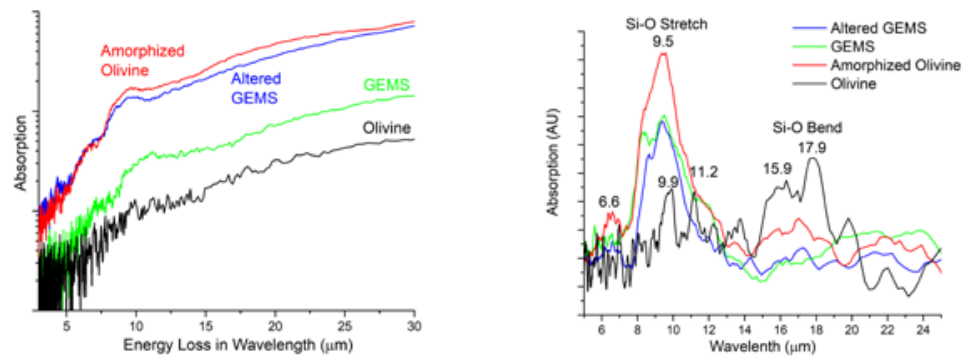

Figure 3. Raw VEEL spectra on a wavelength scale (Left) and after background subtraction (Right). 